\title{
Aspetti geodinamici della diga dell' Ambiesta
}

\author{
P. CALOT
}

Ricevuto l'II Marzo 1964

Rrassuxto. - - Si esamina un anno di registrazioni clinografiehe, ottenute nel roncio centrale della diga dell'Ambiesta, affluente di destra del Targliamento, presso Tolnezzo. Si riassmono orli aspetti linamici fondamentali, che caratterizzano un anno normale di interazione fra lo slarra. mento e le cause perturbanti, con particolare riguardo alie variazioni di livello del lacino idrico (invaso), della temperatura dell aria e della insolazione. Si accenna inoltre alle influenze stagionali sullandamento della rerticale apparente della diga, quale vengono rivelate dalle registrazioni di due coppie di clinografi, sistenate in roccia, ai lati della diga, alla quota del coronamento. Si accenna infine alle variazioni, nel tempo, delle caratteristiche elastiche del calcestruzzo, come vengono chiaramente indicate dalle registrazioni elinografiche di anno in anno.

SUMmar. - We analyse a year of clinographie recordings oltained in the central cut stone of the dam of the Amluesta, right trilutary of the Taglianento, near Tolmezzo. The paper resumes the fundamental dynamic aspects which are peculiar to a normal year of interaction letween the larrage and the perturbing causes, with particular regard to the level rariation of the basin (invaled), of the temperature and the insolation. We also mention the season influences on the hehaviour of the apparent restical of the recordings of two couples of clinographs, set in the rock on the dam sides, at the raffarel altitude. Moreover we report the rariations, in time, of the elastic features of the beton as they are clearly shown by the clinographic recordings, vear l,y year.

Ricerche particolari, sismico-clinografiche, sono state - e sono tuttora - dedicate (1) alla diga sull'Ambiesta, affluente di destra del fimme Tagriamento, nei pressi di Tolnezzo.

Si tratta di una diga di media altezza - concio centrale di $56 \mathrm{~m}-$. costruita verso il 1950. Le indagrini condotte nella zona d'imposta, non si sono limitate alle caratteristiche elastiche della roceia, ma sono state estese anche alle condizioni di cquilibio tettonico delle stratificazioni 
superficiali. Costruita la diuga, il controllo e stato esteso anche al calcestruzzo, sia disponendo tre coppie di clinografi a quote diverse, sul concio centrale, e due coppie in roccia ai lati del coronamento, sia eseguendo periodiche indagini vibrografiche sulla roccia e sul calcestruzzo.

I risultati finora raggiunti - di cui è detto, piu partitamente, in pubblicazioni separate -, riguardano sopra tutto:

I - Comportamento della diga alle azioni provenienti dall'invaso, e dalle sue variazioni.

II - Comportamento della diga all'azione termica, diurna e stagionale.

III - Variazione nel tompo dell'azione termica d'insolazione diurna e sua relazione con la variazione della rigidita del calcestruzzo.

Per quanto concerne il punto I, come si e ottenuto per altre dighe, anche nella diga dell'Ambiesta l'effetto delle variazioni dell'invaso si manifesta dal coronamento alla base della diga, come se il mezzo fosse rigido. Durante la maggior parte dell'anno cio non appare molto distintamente, in quanto - specie per quanto afferisce al coronamento e, sotto certi aspetti, al tampone - e prevalente l'azione dell'insolazione termica. Il fatto però si rende manifesto nei mesi invernali, in cui si va attenuando - per quindi annullarsi - l'effetto dell'onda diurna, con particolare riguardo alla base del concio centrale.

Sempre con riferimento all'invaso va sottolineata, per quasta diga, una particolarita, gia messa in evidenza altrove $\left({ }^{3}\right)$. Quando l'invaso supera un certo limite, la rotazione verso valle viene soviastata, con particolare riguardo al coronamento, dal raddrizzamento che subisce la parte superiore del manufatto, come conseguenza di una maggiore flessione nei confronti del coronamento stesso.

Passiamo al punto II.

L'azione flettente dell'onda diurna, come avviene per altre dighe, diminuisce dal coronamento alla base del concio, dove questi confina col tampone ad agisce quasi esclusivamente sulla componente vallemonte. Mediamente, in questa - come in altre dighe - l'andamento della flessione dal coronamento alla base del concio centrale e rappresentato dalla Fig. 3, dove M rappresenta il coronamento e $\mathrm{O}$ il tampone; alla base del concio, la flessione è pressoche nulla $\left({ }^{4}\right)$.

Il tampone ha un comportamento a se; l'onda diurna si manifestava. qui su entrambe le componenti - sebbene, in modo preponderante e, a prima vista, inesplicabile, sulla componente destra-sinistra. Va peró 


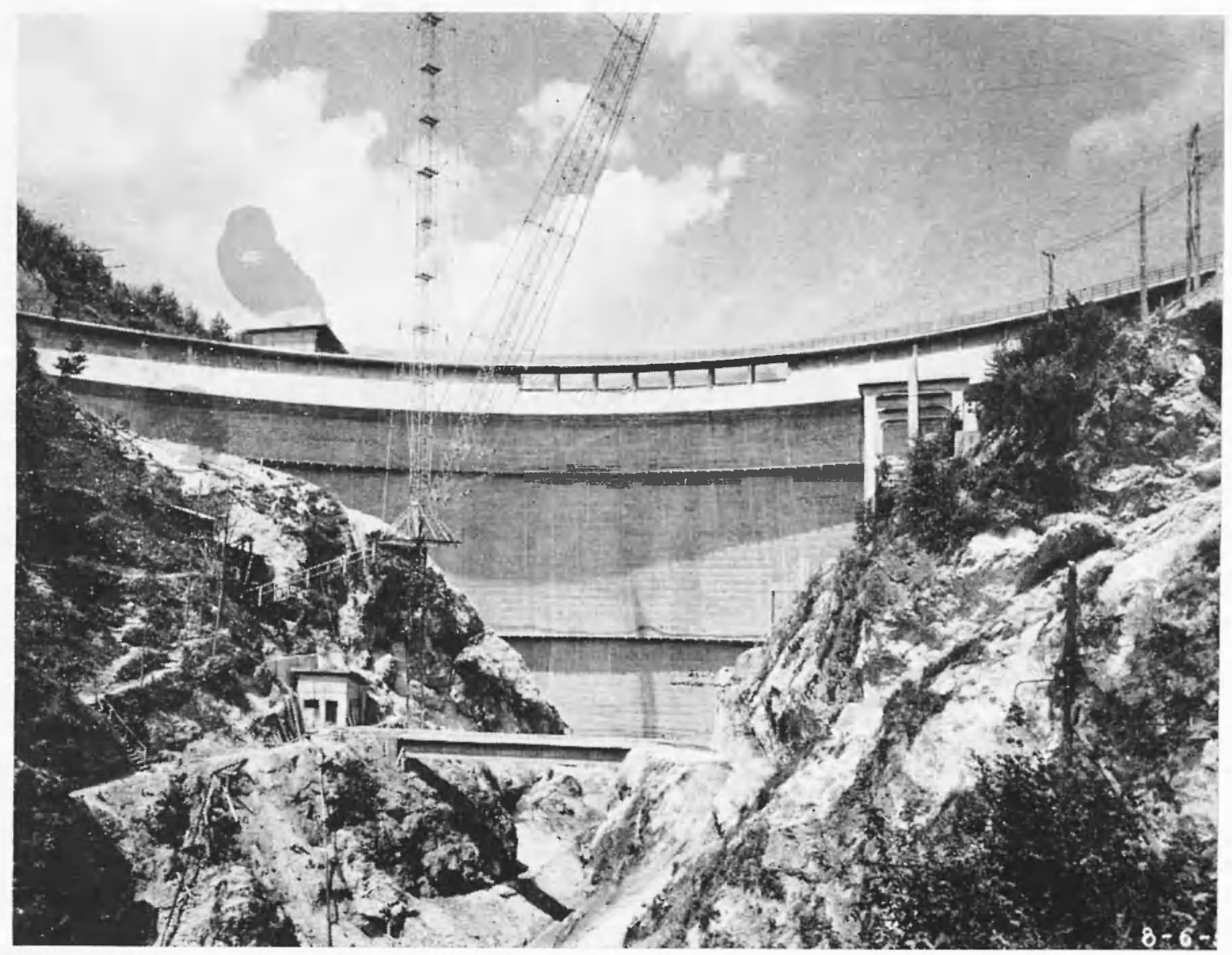

Fig. 1 - Diga dell Aubiesta, vista da valle. Nel coneio centrale funzionano tre copplie di olinograti. Lat copplia del coronamento e sistematil nel enuicolo, in compispondenza della luese rentrale del ponte. Le altre due eopplie sono sistemate alla base del concio (9. 437) e sul tampone. un metro sotto (q. +36), nella casetta custodia ripmodotta nella Fig. 2. 


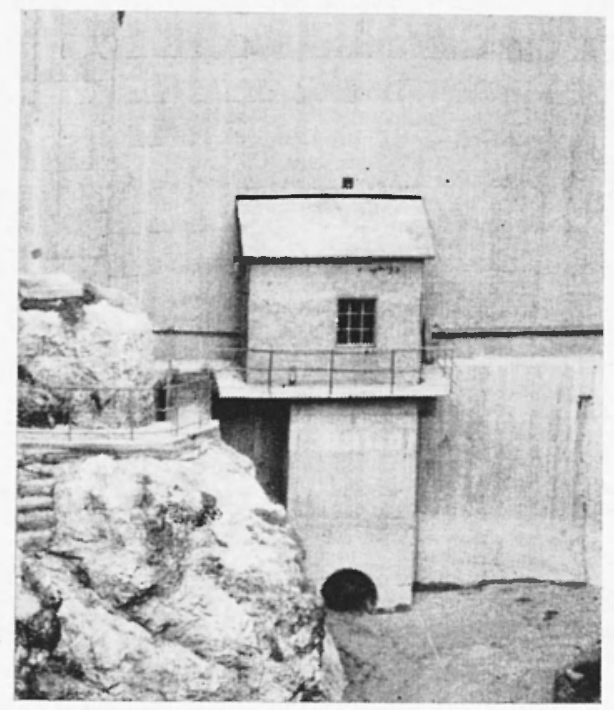

Fig. 2 - Casetta custodia dei clinografi alla base del concio centrale e sul tampone.

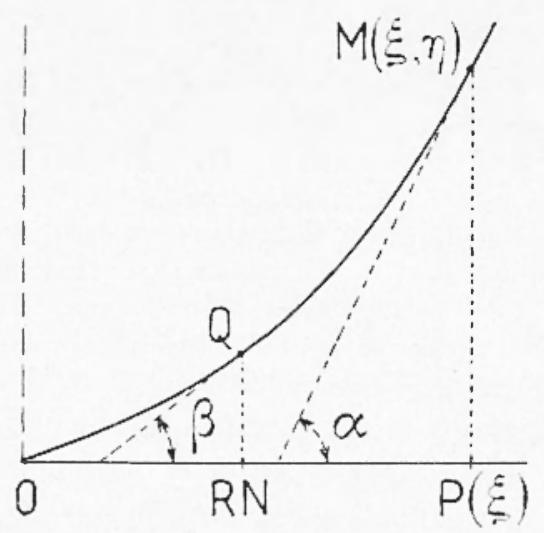

Fig : 

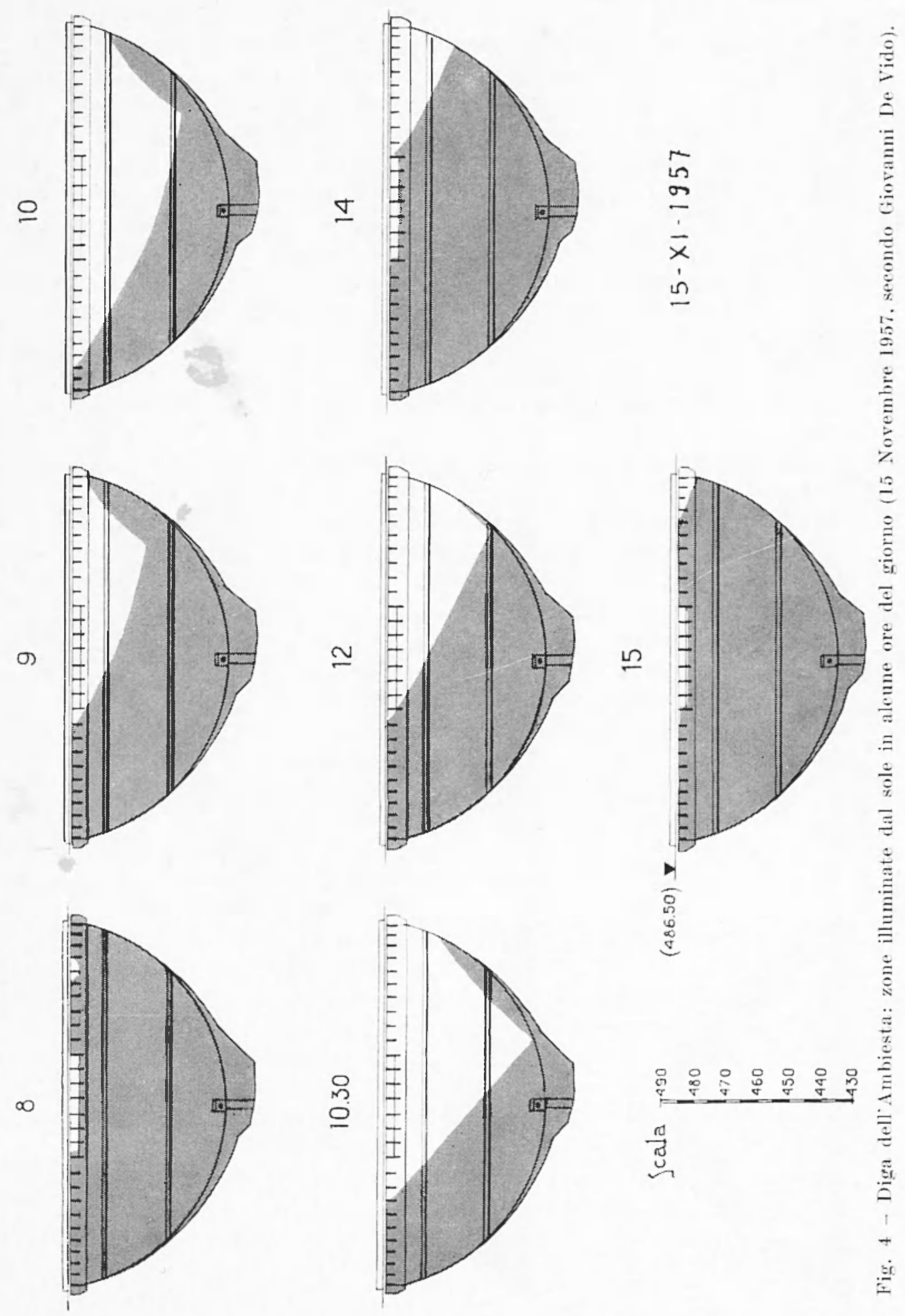

10 

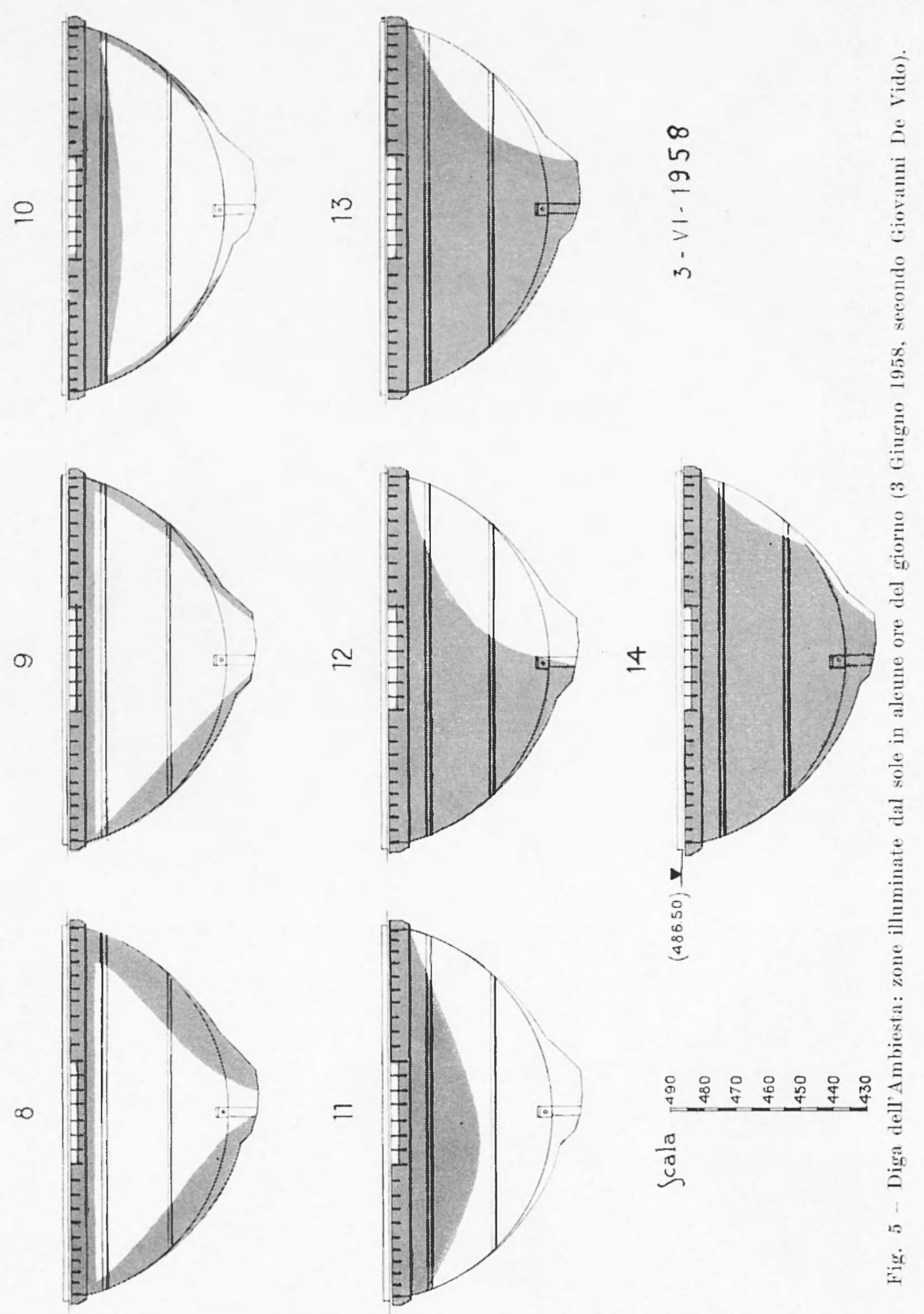
fatta una precisazione. Isa componente valle-monte non va piu attribuita alla dilatazione trasrersa del concio: si a visto, infatti, che tale causa diminuisce di efficienza dal coronamento rerso la base dol concio, dove praticamente si annulla. Essa deriva invece dallallungamento del concio sovastante per ditalazione, e poiche il riscaldamento eresee rerso laalto della diga, cio provoca un innalzamento del centro di gravita del conceio stesso, con conseguente " caduta" verso monte; in fase di rafiredramento, si verilichera il fenomeno contrario. L'asimmetria della diga all'insolazione a invece la causa della notevole componente a novanta gradi (sinistra-destra), tangenziale allaasse della diga. Poiche l’insolazione agisce, in maniera preponderante, sul lato sinistro della diga (Figg. 4-5̃), riesce massina la sua azione - in fase positiva - verso tale lato, mentre durante la notte la contrazione del calcestruzzo riportera la verticale a)parente verso destra.

('io spiega in modo chiaro, la presenza di un'ampia onda diurna sul tampone, sulla componente destra-sinistra, $u n$ solo motro sotto la postazione rigidamente comnessa alla base del concio centrale, dove l'onda diurna e pressorlie nulla, come risulta dalle Figg. da 8 a 15 . Isil componente valle-monte registra ivi soltanto le variazioni di flessione associate alle valiazioni d'invaso (le quali lanno pure, generalmente, periodicita diurna, e provocano movimenti langamente in fase con quelli dorigine termica, roi quali, ad ogni modo, non vanno confusi), che non impressionano affatto (o in modo trasculabile) la componente tangenziale spalla-spalla la quale - come si e visto - un metro piì sotto a fortemente influenzata dall'asimmetrica azione dinamicar dell'insolazione.

Quanto e stato detto, vale naturalnente durante i mesi in cui l'azione dell'insolazione riesce efficance. A partire dal Novembre a lino alla finc di Febbraio, tale azione riesce pressoche nulla (come presso alt re dighe) a londa diuma effettivamente seompare, anche in quota. Di conseguenza, scompare pure il singolare fenomeno d'asimmetria dinamica diuma, di cui il tampone è sede negli altri mesi, e che ricompare chiaramente, invece, rerso la fine di Febbraio (Fig. 7).

A prima vista, potra sembrare difficile ammettere un"indipendenza di movimento fra il tampone e le basi dei conci sovastanti, che risultano, allatto pratico, formare un unico bloceo. Son ra pero dimenticato che i movimenti in questione sono in realta micromorimenti, e che il tampone e effettivamente sepalato dalle basi dei conci da superficie di discontinuita meceanica, che rendono possibili tali movimenti infinitesimi. Quando questi moti divengono finiti, la diga toma a reagire come un tutto unico (almeno, in prina approssimazione). 


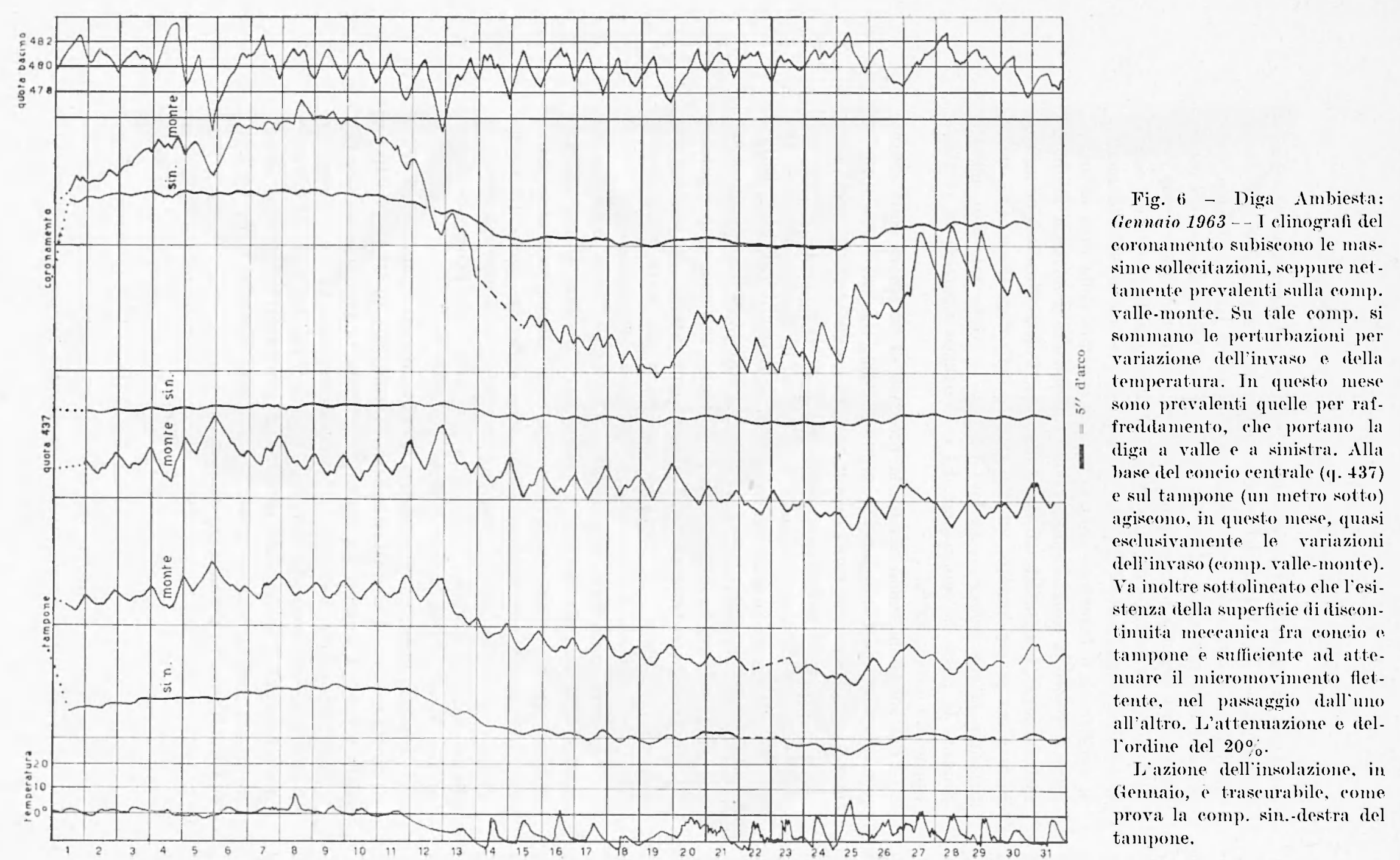




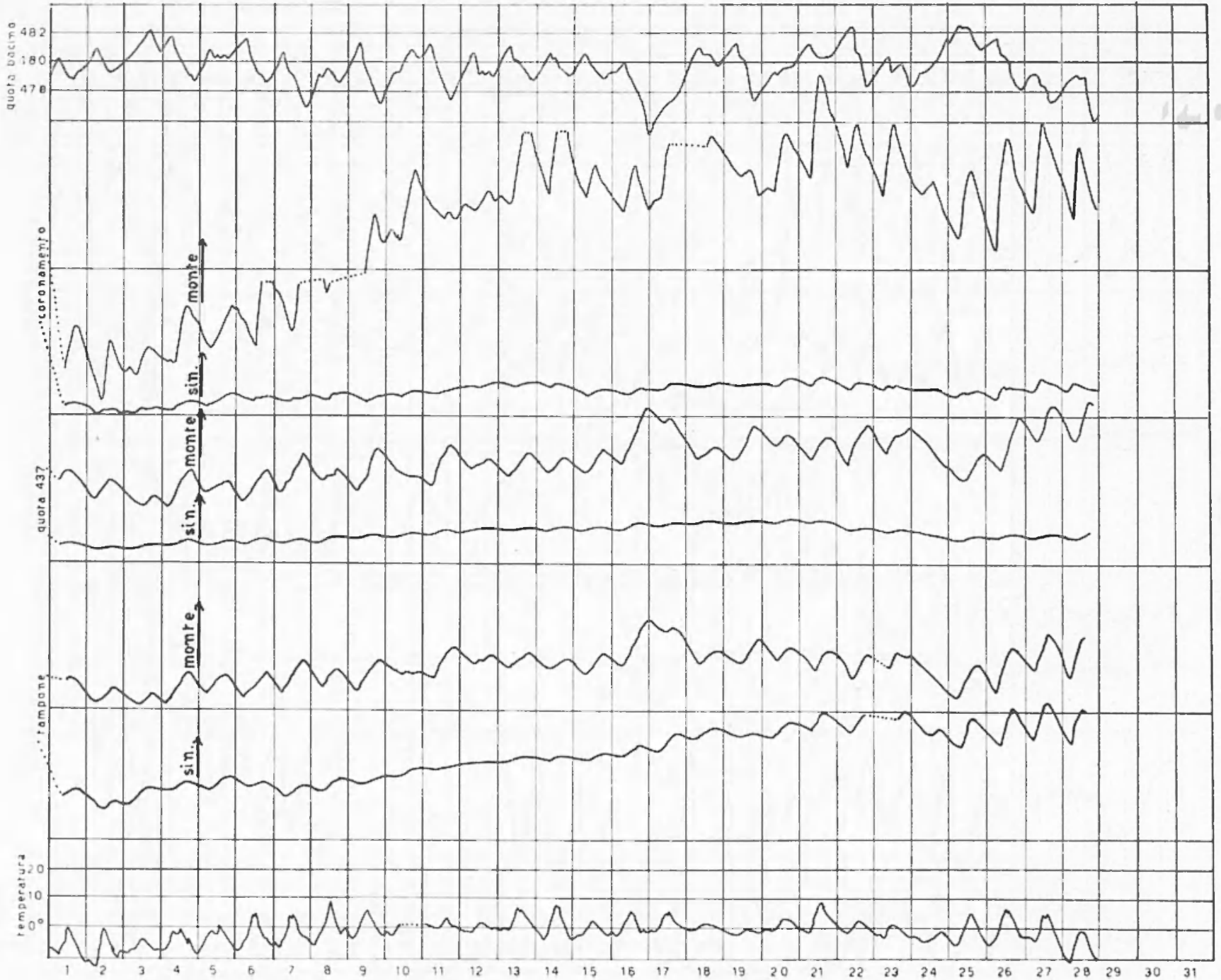

Pig. 7 - Diga Ambiesta: febbroio 1963 - II graduale a umento della temperat ura media dell aria, provoea linversione del movimento di rotazione della diga, ehe orat tende verso monte, cont ampiezza aceentuala nel coronamento (comp. valle-monte). Le componenti valle-monte a quota 437 a 436 (1:ampone), rispecchiano, sostanzialmente, lan. damento dellinvaso. L'azione asimmetried dell'insolazione comineia a farsi sentire sulla comp. destra-sinistra del tam. pone, dove, verso la fine del mese, riappare netla londa diurna (gia cevilente sulla comp). valle-monte del coronanento). 


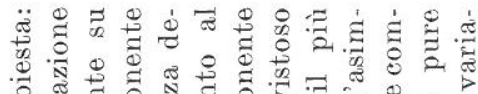

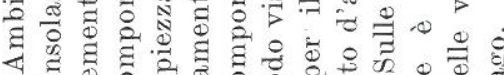

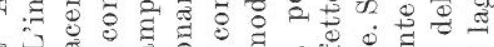

䑻|

๗

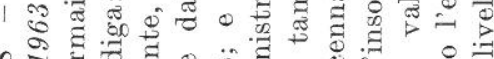

0

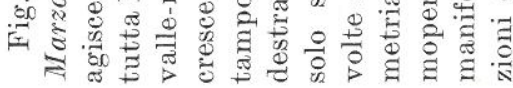

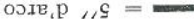

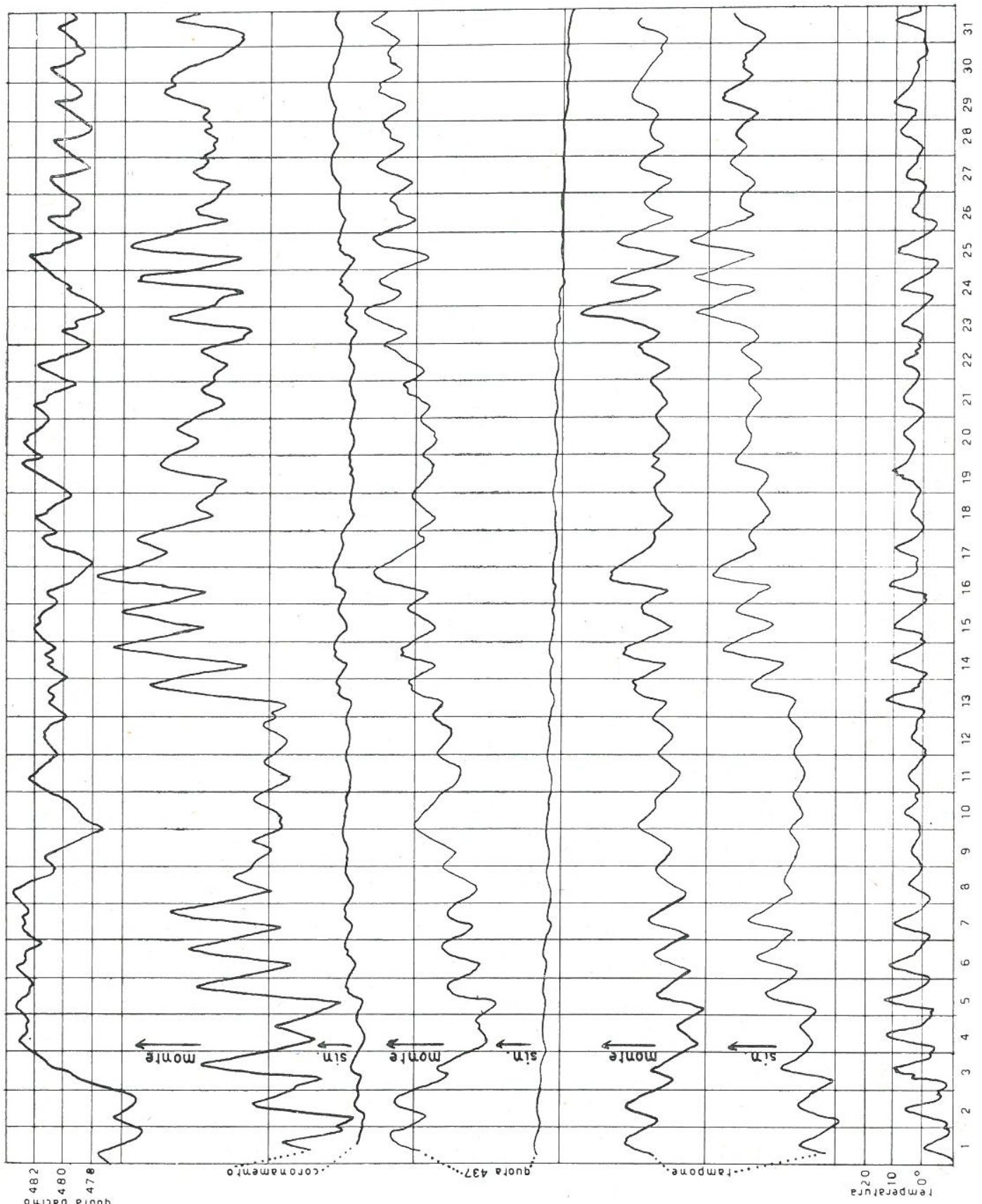




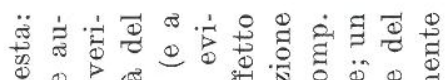

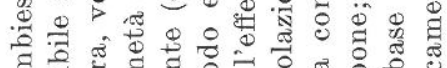

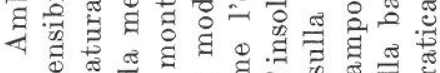
और

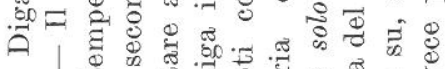
๓

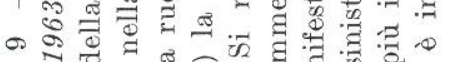

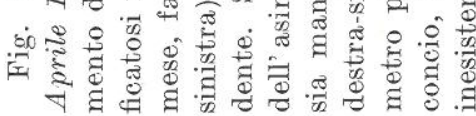

oगIe,p, $\mathrm{S}=$

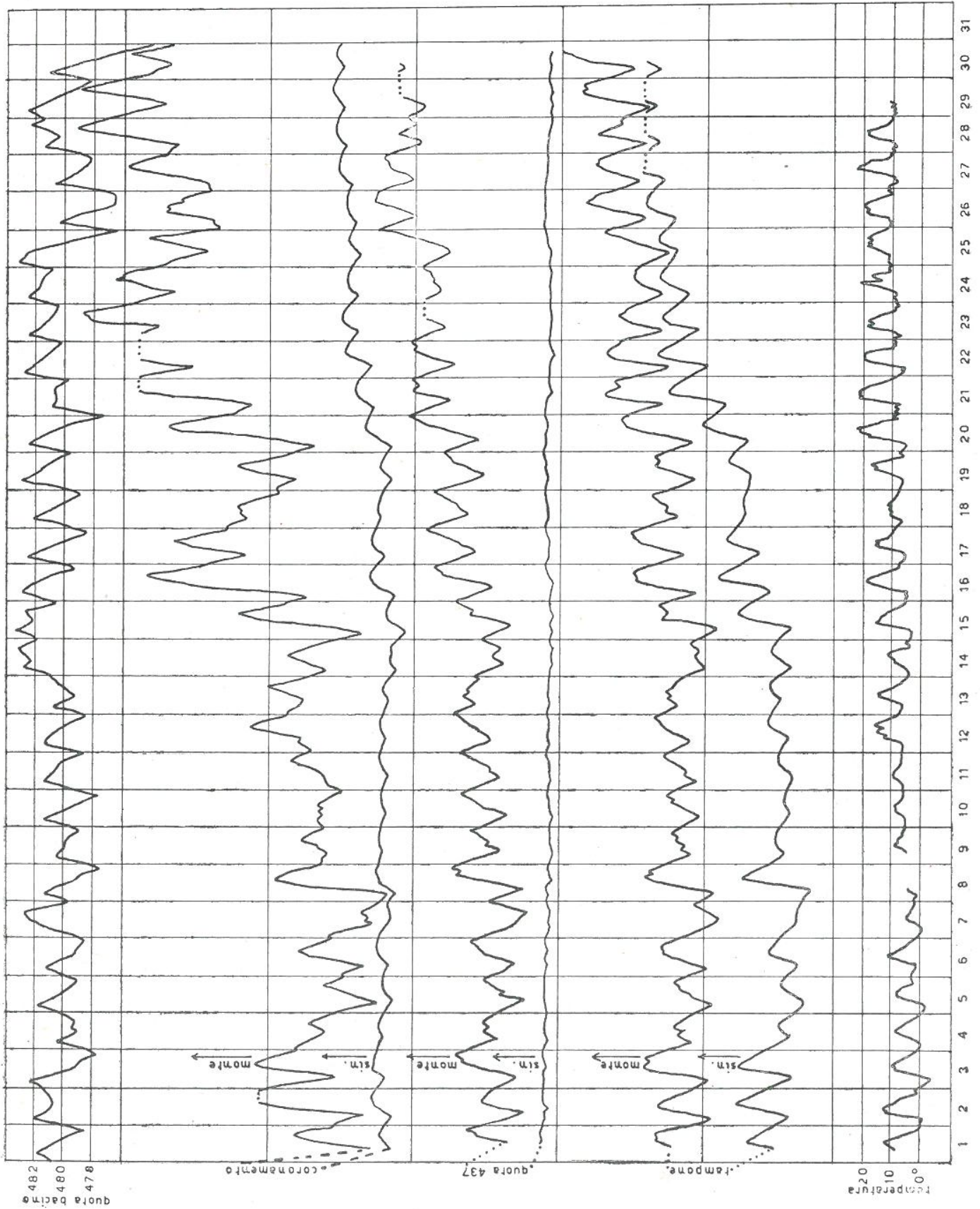




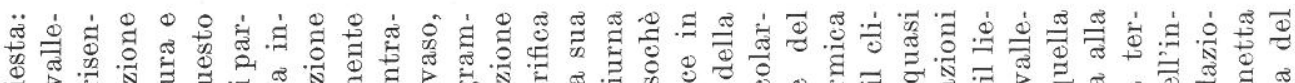

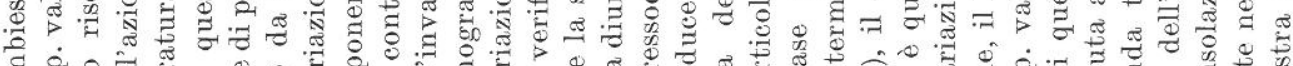

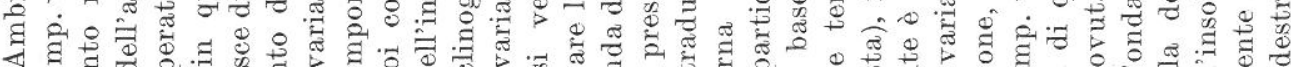
य

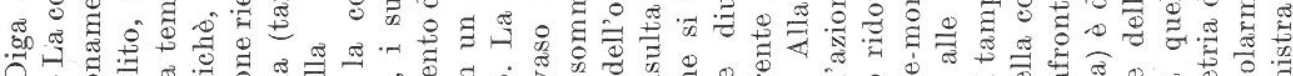

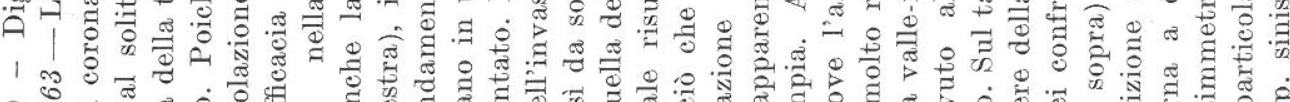

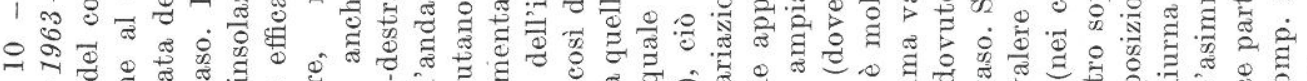

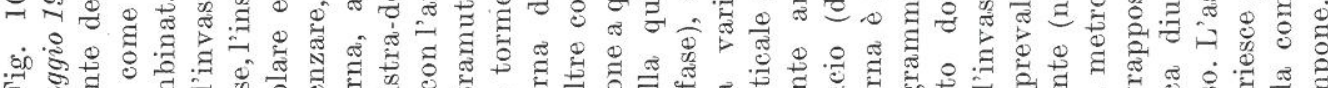

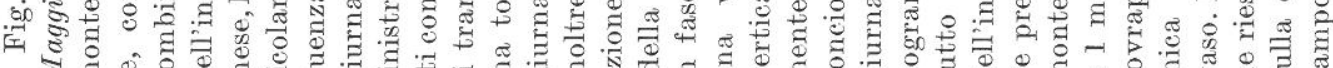

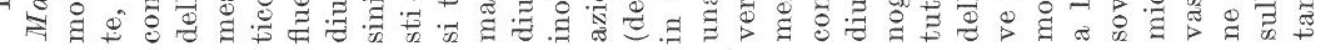

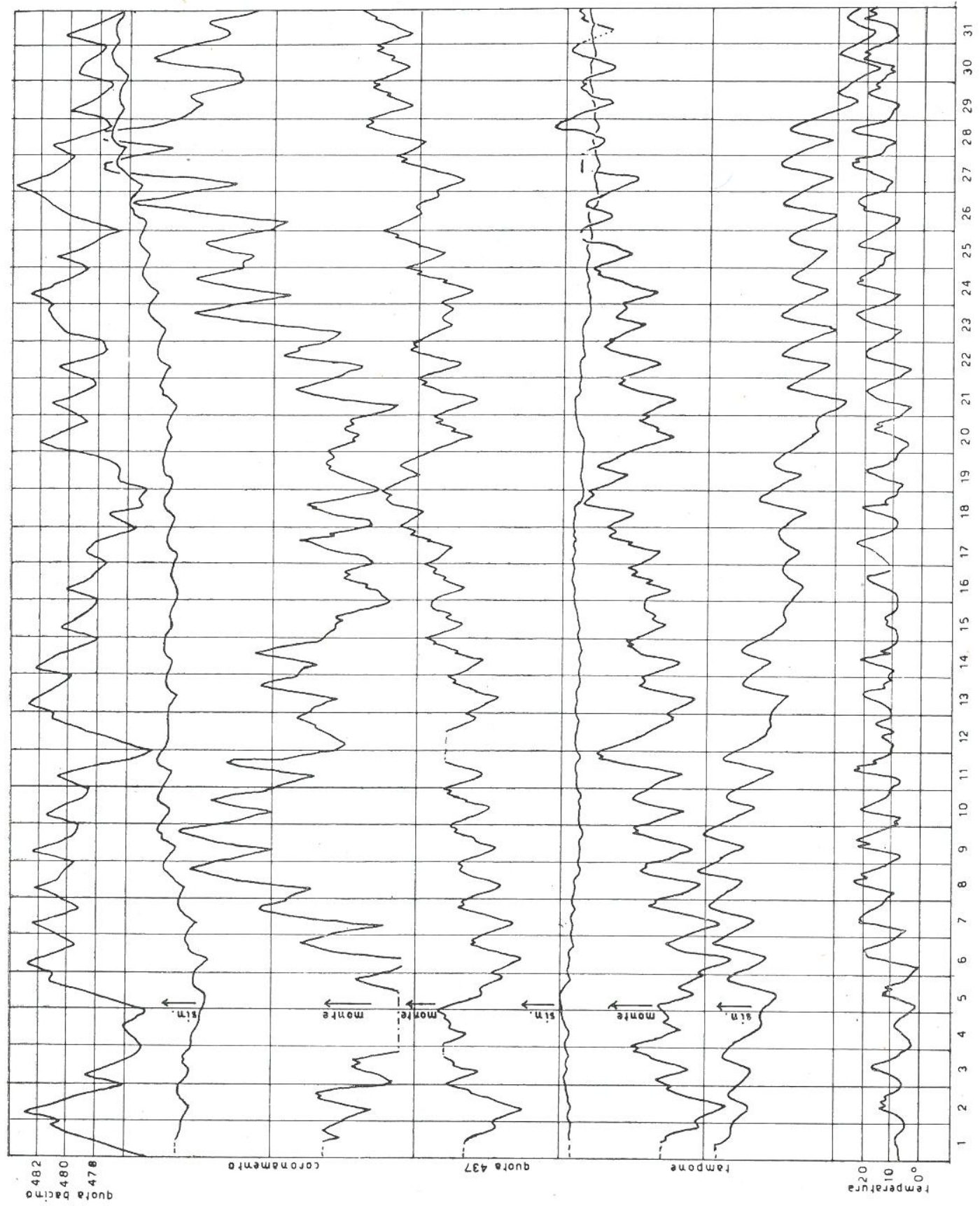




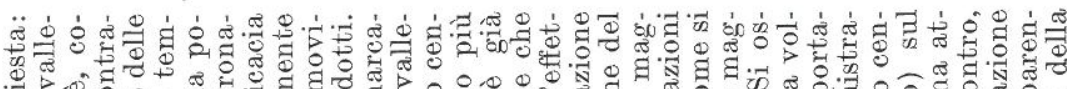

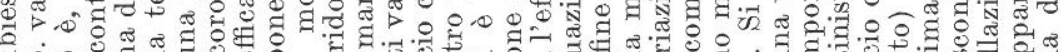

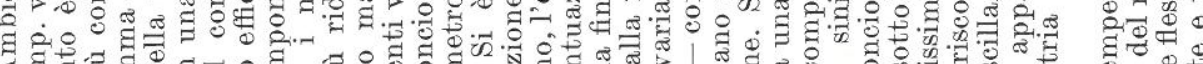
《 छ ऽ

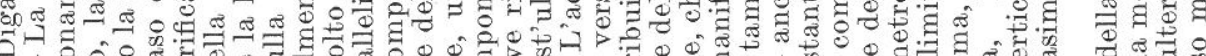
A

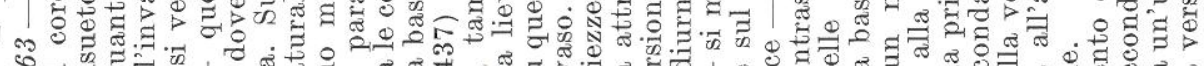
-

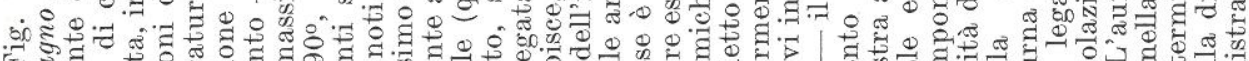
प.

orle,p $/, \mathrm{S}=$

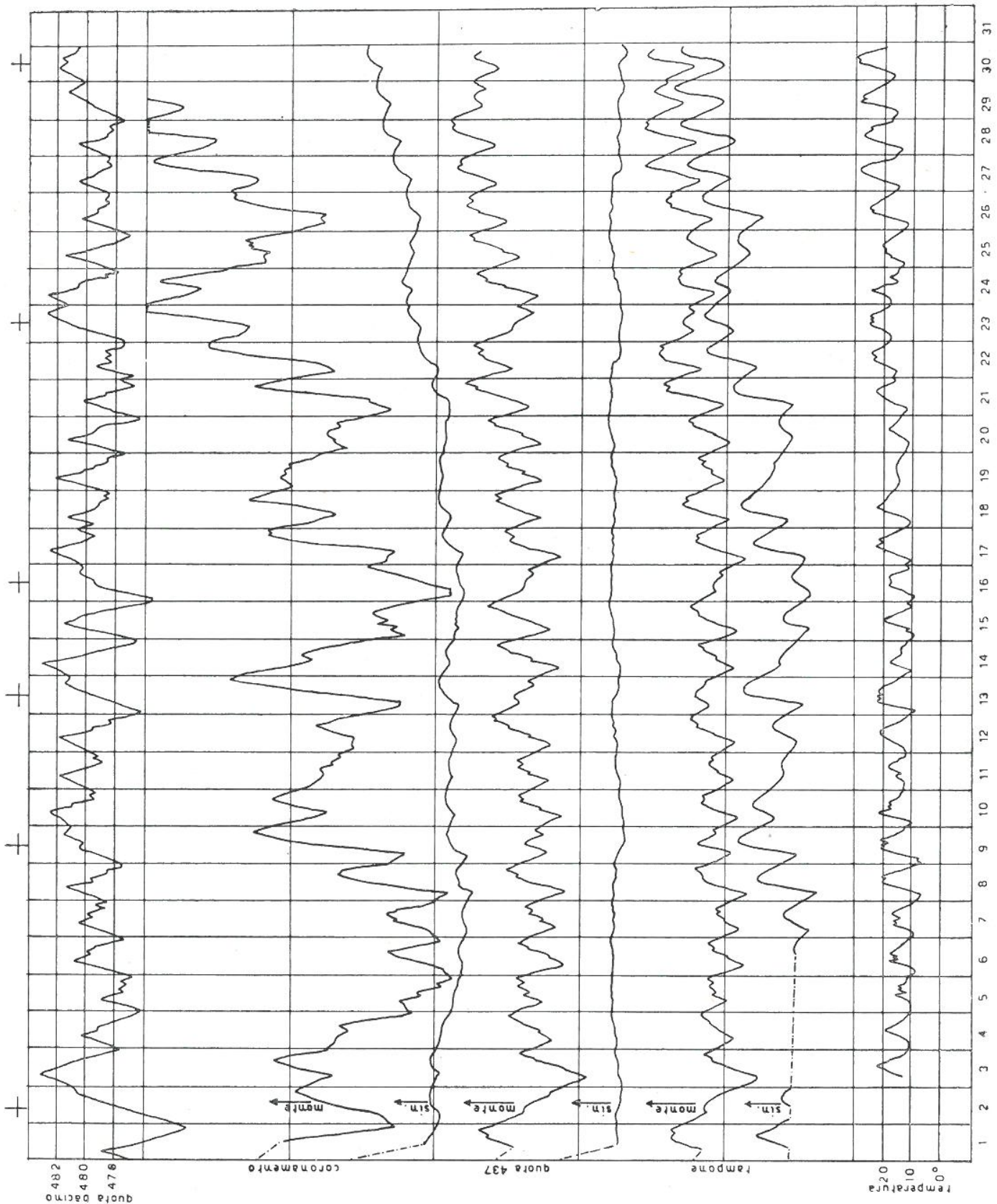




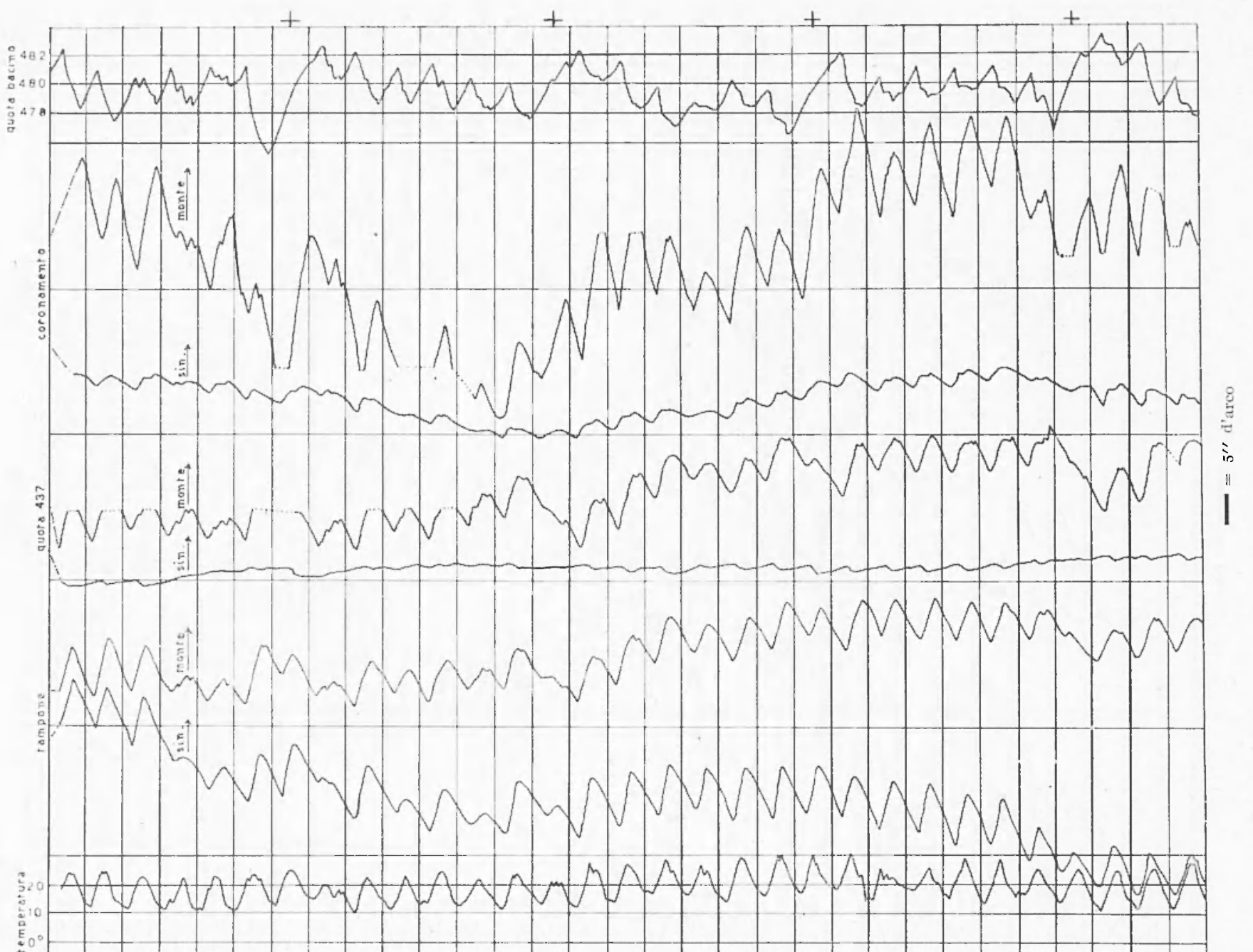




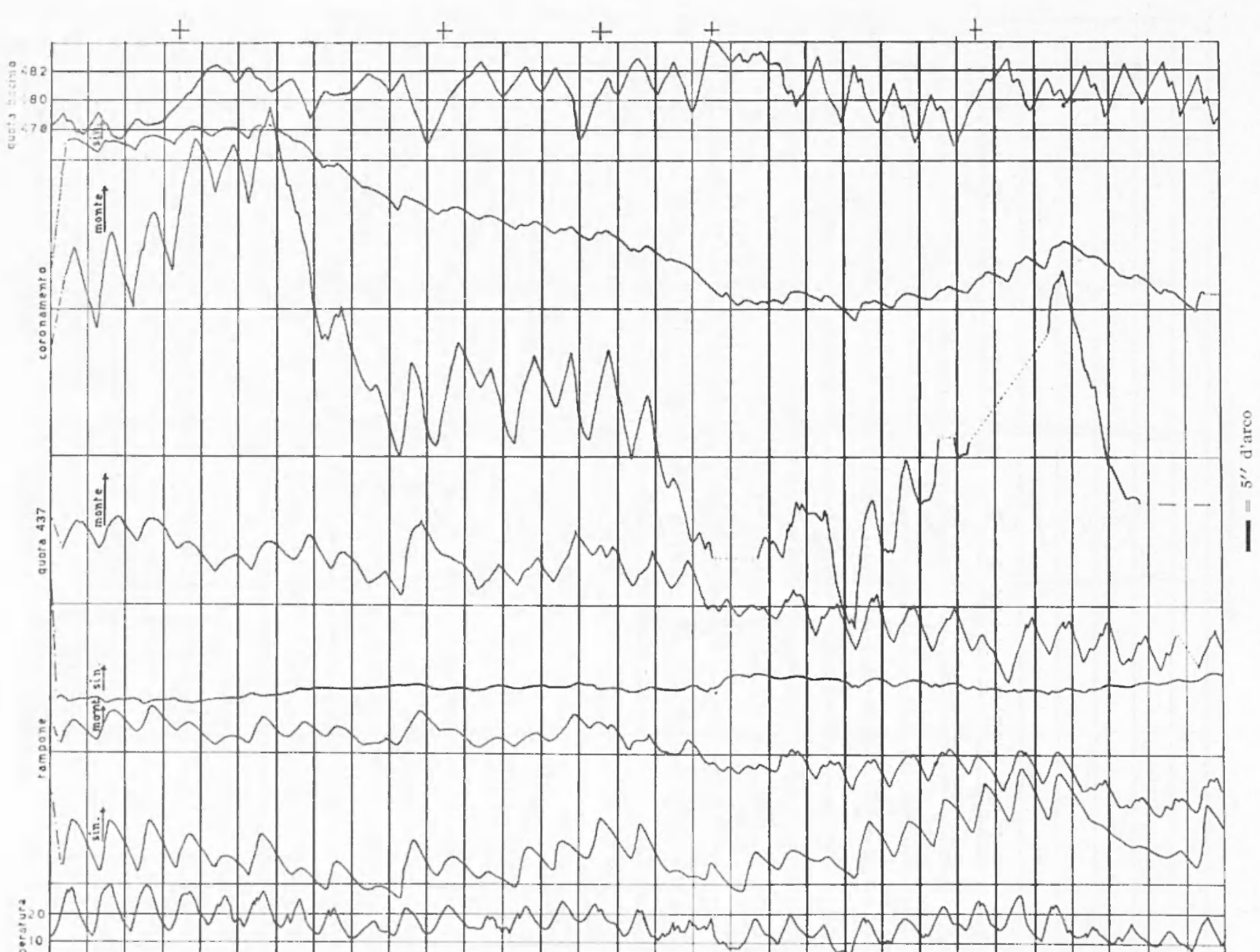


orde, $\mathrm{p} / \mathrm{s}=$

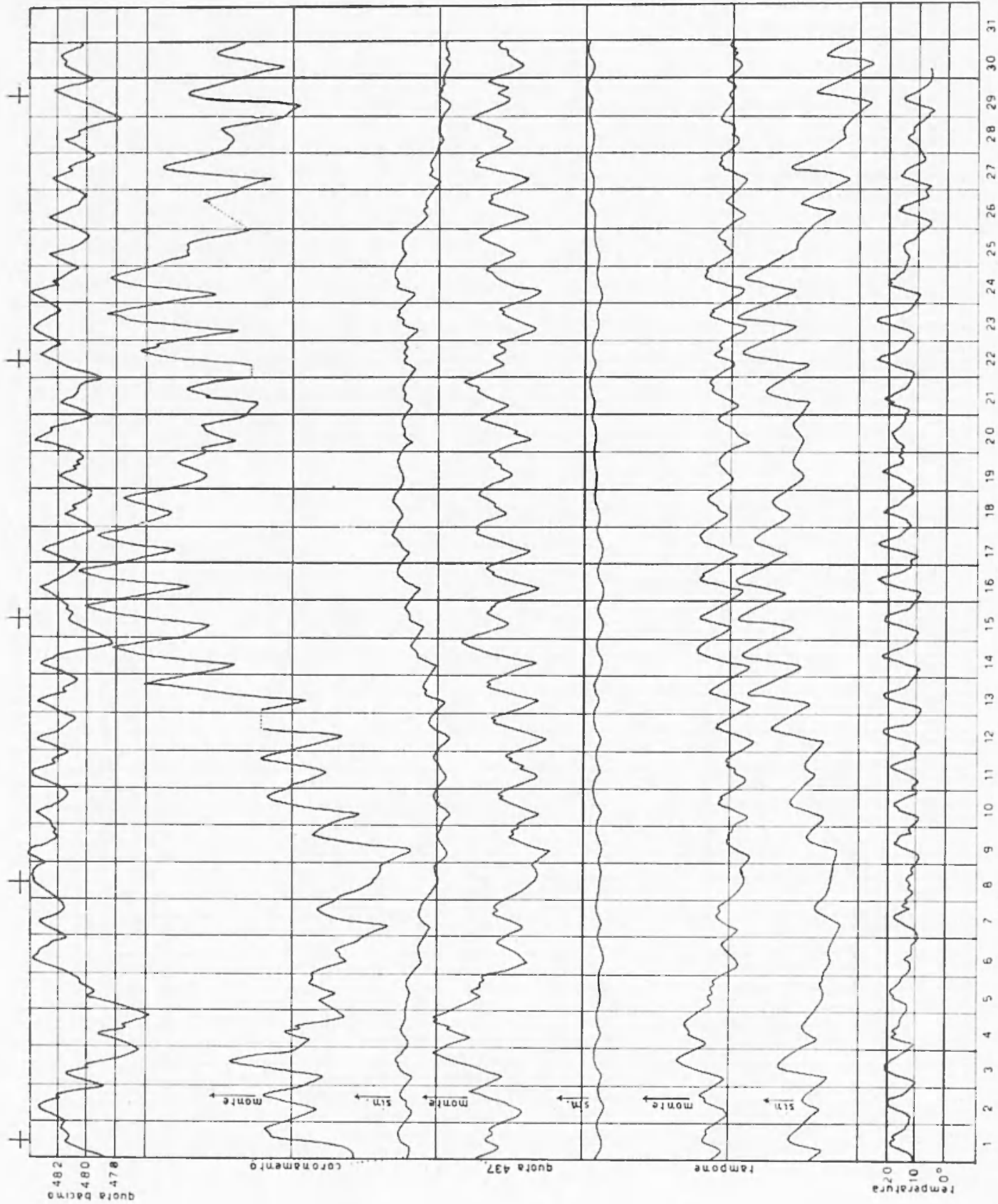




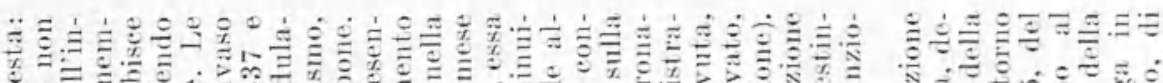

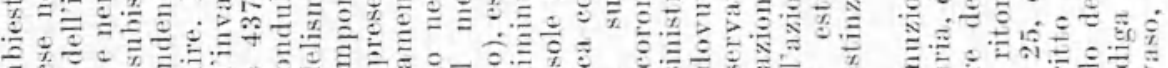

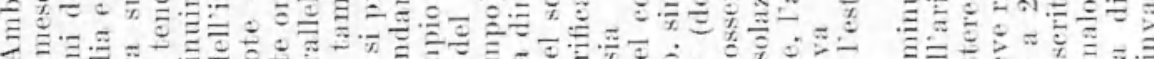

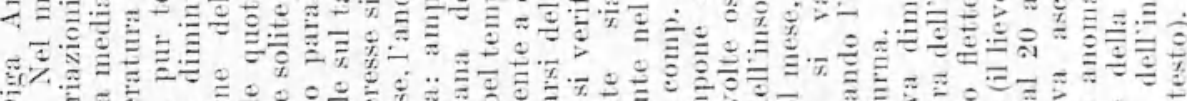
并

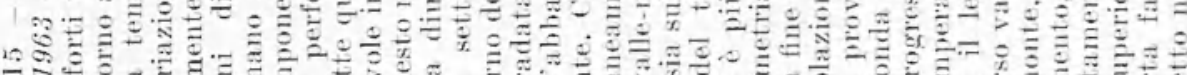
a

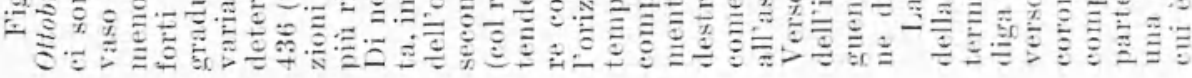

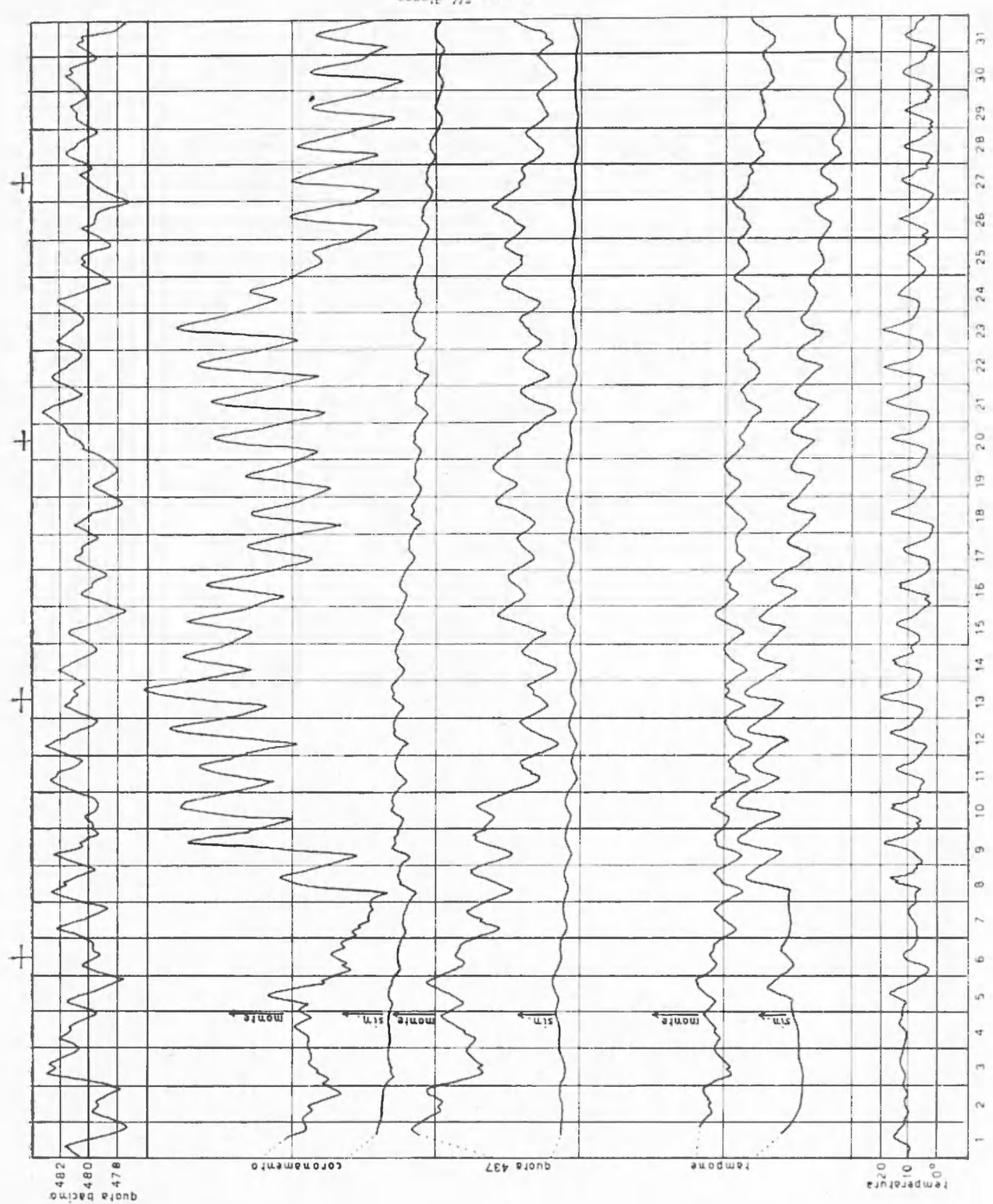




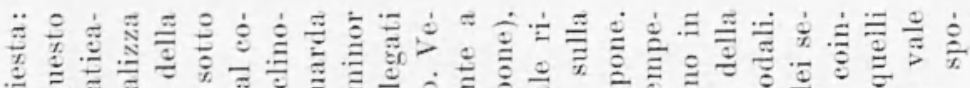

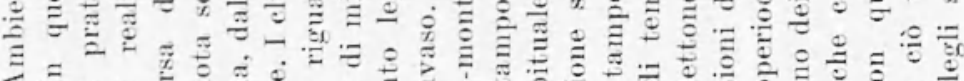

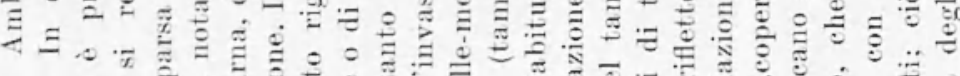
幽|

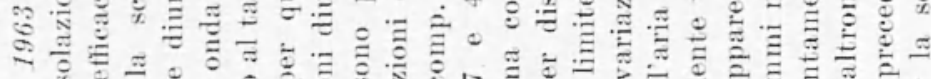

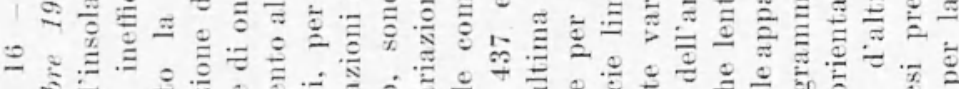
๘

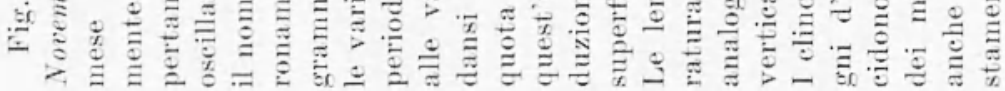

ore, $, \mathrm{S},=$

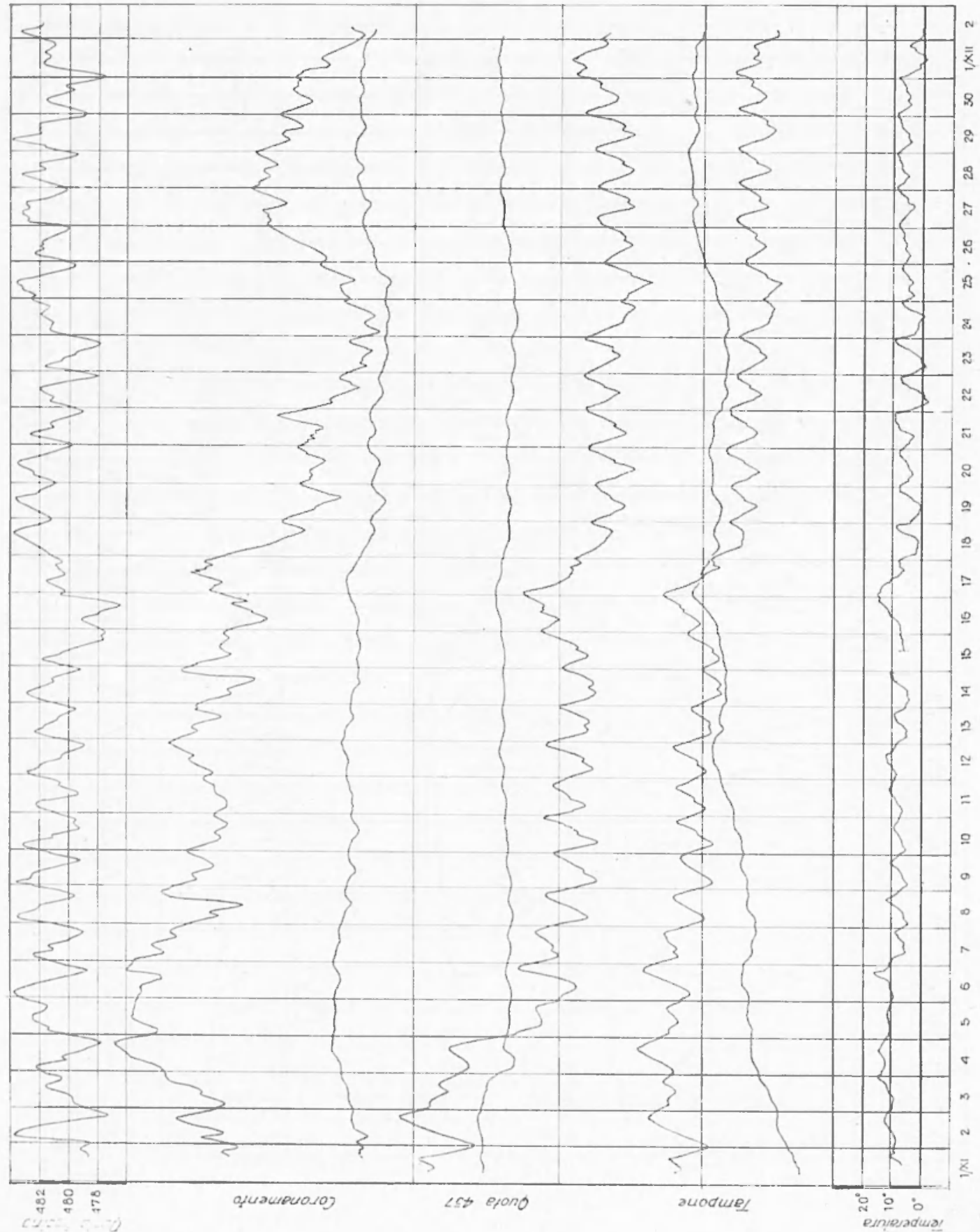


os. $, \mathrm{p}, \mathrm{S}=$

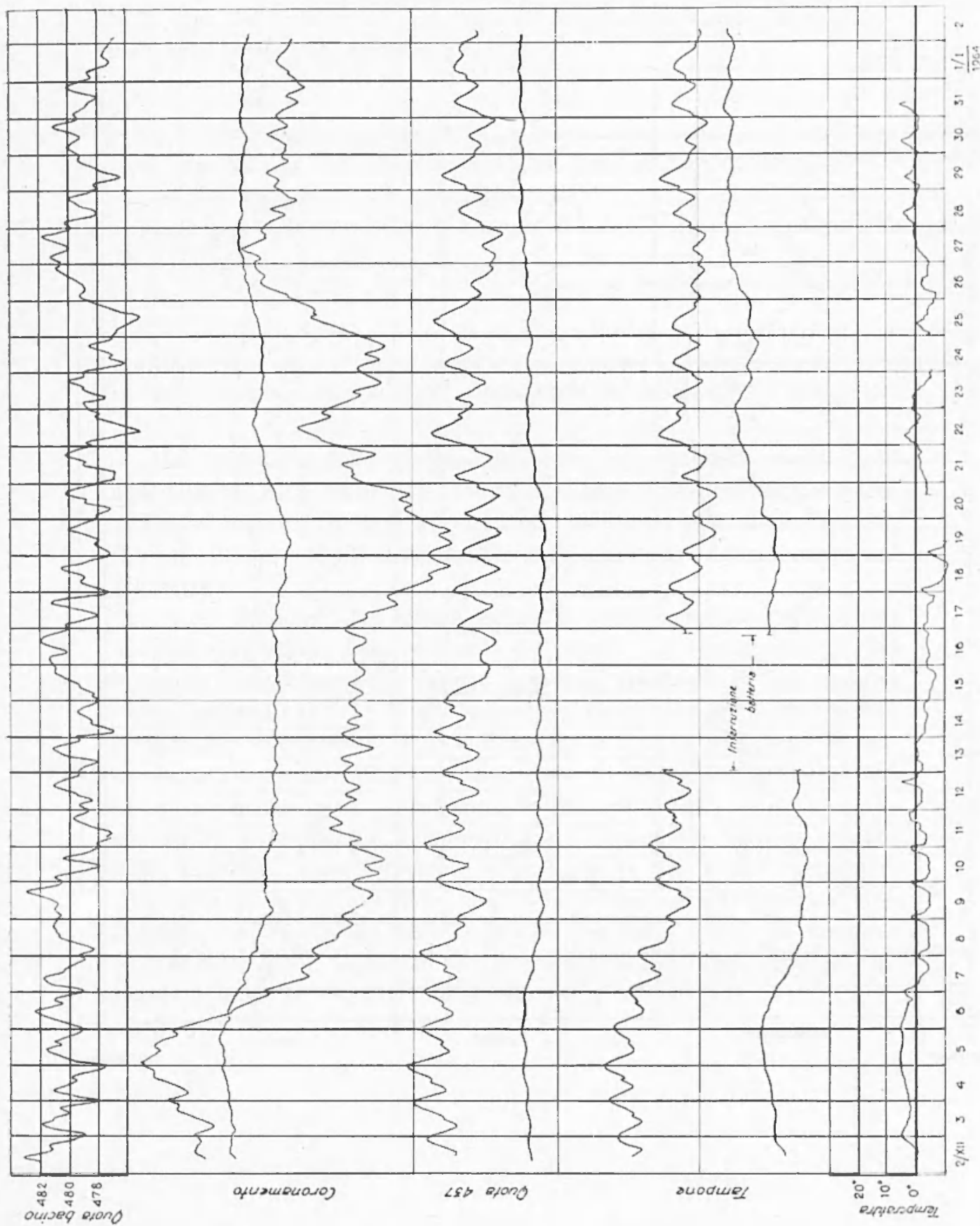




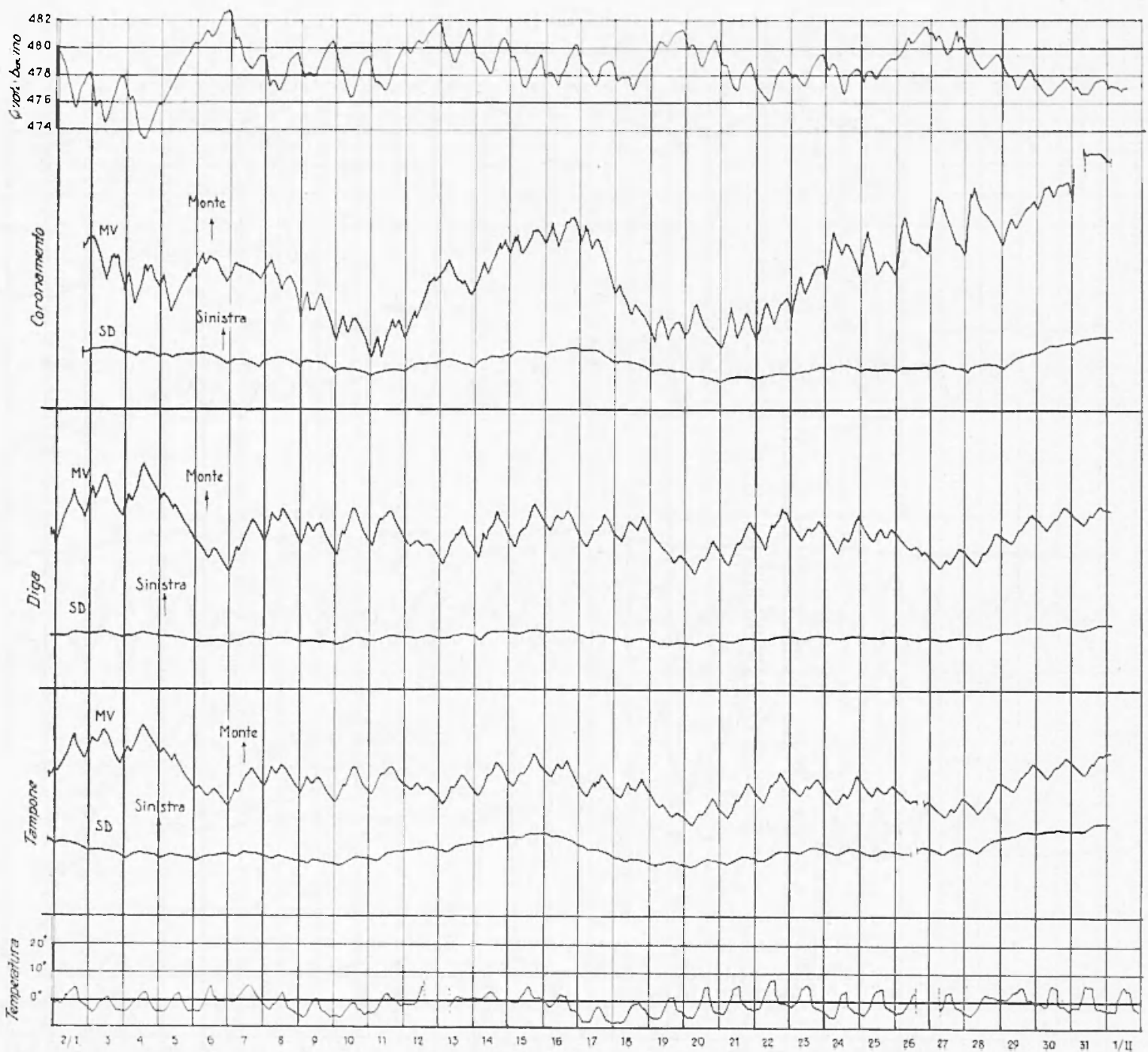

Fig. 18 - Diga Ambiesta: Gemaio 196t - Quello che si e detto per Novembre e Dicemhre, vale sostanzialmente anche per fentuaio: per i motivi piu volte esposti, solo le eomponenti valle-monte somo attive per le variazioni della verticale apparente, di periodiciti uguale o minore a lun giomo, attribuibili alle sole variazioni di livello nel bacino idrico. Manca l'onda diurna e solo le lente variazioni di temperatura dellaria agiscono, in questo mese, sulla diga. 
Venendo al punto III, possiamo osservare che i fenomeni aceenmati al punto II subiscono, a loro volta, variazioni non periodiche nel tempo, legate alle variazioni della natura intrinseca del calcestruzzo. Cio si manifesta sia per decadimento dell'elasticita per trizione della roceia d'imposta continuamente sollecitata, sia per mutata rigidita del calcestruzzo. Io gial mostrato altrove come tale mutazione possa essere messa in evidenza dal controllo periodico delle frequenze libere dei conci della diga. Tale metodo ha gia provato l'aumento della rigidita p. es. nella diga del Lumiei, ed è in corso di applicazione presso altre dighe, compresa quella dell'Ambiesta (Fig. 19).

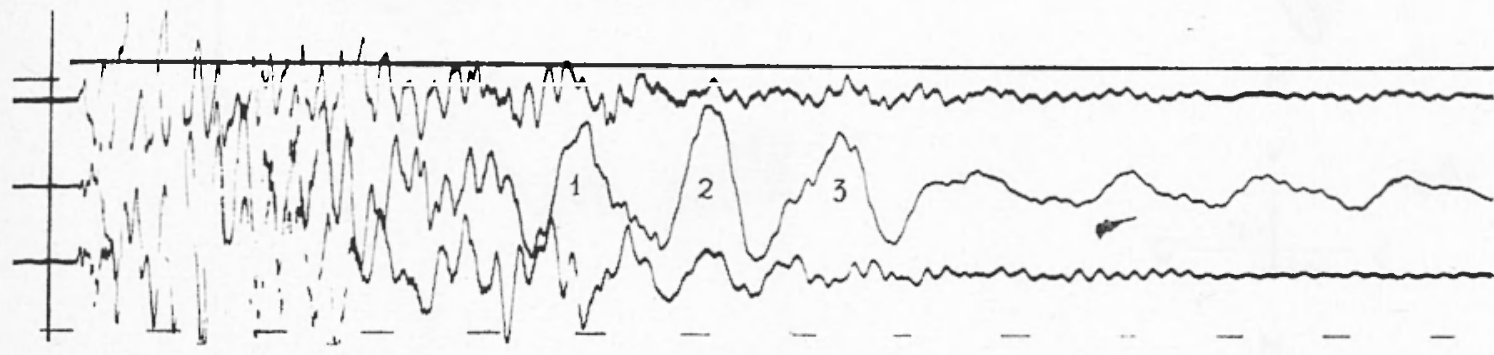

Fig. 19 - L'oscillazione 1-2, 2-3, sulla componente orizzontale valle-monte del vibrografo da la fondamentale trasversale del concio centrale (periodo os, 18 circa).

La variazione della ligidta viene pure espressa dalle registrazioni clinografiche. Si e detto $(2,4,5)$ dell'attenuazione e successiva scomparsa dell'onda diuma alla base di una diga, dalla sua ultimazione in poi. Cio puo essere senz'altro spiegato con il progressivo irrigidimento del calcestruzzo.

A tale fenomeno, va pure associata la lenta variazione del piano di ondulazione diurna della verticale apparente, in corrispondenza del tampone della diga dell'Ambiesta. Il vettore risultante di tale ondulazione, negli anni $195 \tilde{5}-58\left({ }^{3}\right)$, presentava un'inclinazione di $30-40^{\circ}$ rispet to la direzione valle-monte. Tale inclinazione e andata gradatamente aumentando, tanto che nel 1962 essa e stata, in media, dell'ordine di $60^{\circ}$. Cio sta a significare che - a parita di altre condizioni — e andata gradualmente aumentando la resistenza del mezzo alle flessioni causate dalle variazioni termiche, legate all'andamento diurno dell'insolazione. Attualmente, si puo affermare che lazione dinamica, determinata dall'asimmetria dell'insolazione, è la sola causa termica agente sul tampone e si esplica, quasi eschusivamente, sulle variazioni della verticale apparente da spalla destral a spalla sinistra e viceversa (Fig. 20). 
I)ellazione stagionale si a riferito più rolte $(2,3,4,5)$. Essa appare evidente anche ai margini della diga, sui clinografi sistemati fuoli dli essa, a testimonianza dell’influenza dellambiente sul comportamento della digra stessit.
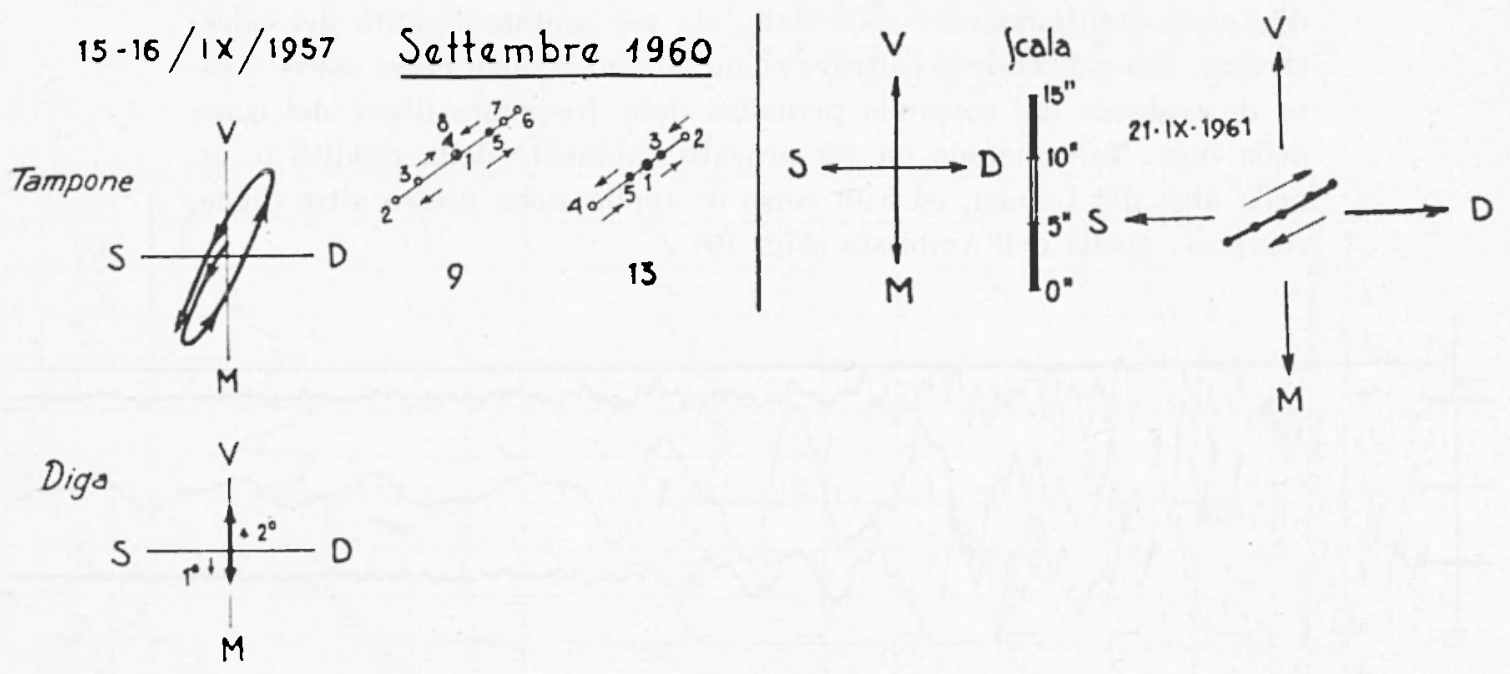

Fig. 20

Fra i rlinografe funzionanti fuori della diga, rivestono particolare interesse, a questo riguado, quelli sistemati in roceria ai lati del coronamento, una roppia in sponda sinistra ed una in sponda destra. Si a

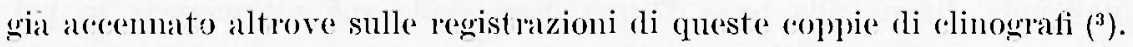

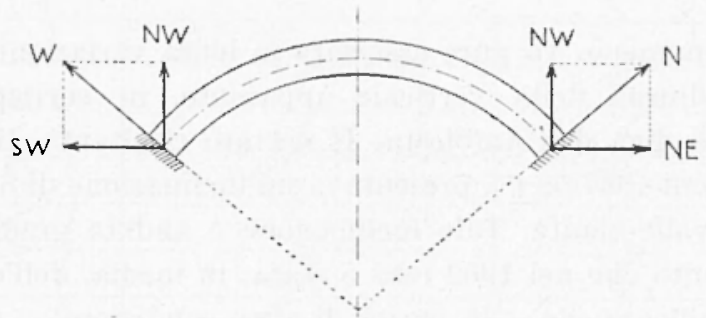

ligr. 21

Qui desidero soflemare l'attenzione sulle indicazioni che essi forniseono rirca la stagionale interazione fra diga e roceia d'imposta.

Si vedano i clinogrammi ottenuti, nelle postazioni dette, nell'anno 1961. İ facile l'interpretaziono dol movimento romplessivo da essi 



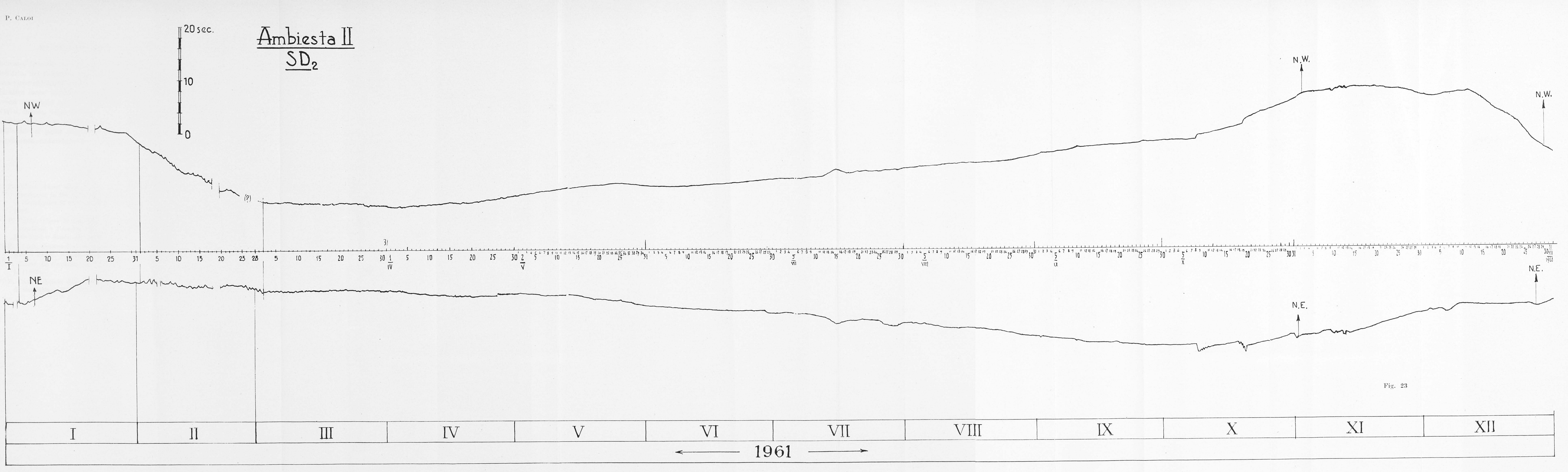


indicato. Quando la diga subisce l'effetto della temperatura crescente dell'aria, nel passaggio dall'inverno alla primavera e, quindi, all'estate, essa viene obbligata a spostarsi verso monte. Mediamente (prescindendo da altre cause di perturbazione) nelle postazioni in sinistra dovranno aversi variazioni angolari verso $\mathrm{NW}$ e $\mathrm{NE}$ e, in destra, ancora verso NW e, invece, verso SW per l'altra componente (Fig. 21). È quello che sostanzialmente si verifica, come i provato dalle Figg. 22, 23. Quando dall'estate si passa gradualmente all'autunno e, quindi all'inverno, il movimento, naturalmente, si inverte.

\section{BIBLIOGRAFIA}

() P. Calor, About some Phenomena preceding and following the seismic Movements in the Zone characterized by high Seismicity, "Contributions in Geophysics in Honor of Beno Gutenberg". Pergamon Press, London, 1958.

(2) P. CaLor, Come la Geofisica può contribuire ai problemi concernenti la costruzione e l'osservazione delle grandi dighe. SADE, Ufficio Studi Relazioni e Studi, 19, 1958.

(3) P. CaLoI, Osservazioni clinografiche presso la diga dell' Ambiesta. SADE Ufficio Studi, Relazioni e studi, 21, 1959.

(4) P. CALoI, La Geofisica e le grandi dighe. "L'Energia Elettrica ", XXXIX, 1, (1962).

(5) P. CALor, Aspetti della dinamica di rocce, calcestruzzo ed acque. "Annali di Geofisica", XV, 2-3, 1962. 